\title{
Performances et conduite des animaux de trait en Afrique sub-saharienne : une synthèse *
}

\author{
R.A. Pearson ${ }^{1}$ E. Vall ${ }^{2}$
}

Mots-clés

Bovin - Ane - Cheval - Animal de trait Evaluation - Aptitude au travail Performance animale - Conduite d'élevage - Afrique au sud du Sahara.

\begin{abstract}
Résumé
L'utilisation de l'énergie animale permet, le plus souvent, aux paysans d'Afrique sub-saharienne d'augmenter leur production agricole et d'améliorer leur qualité de vie. L'utilisation efficiente de l'animal de trait dépend d'une bonne maîtrise de ses capacités de travail, des conditions d'élevage et des facteurs qui peuvent influencer ses performances. Ces aspects sont passés en revue dans cet article dans le contexte de l'utilisation de l'énergie animale pour l'agriculture en Afrique sub-saharienne. L'espèce animale utilisée conditionne la quantité d'énergie disponible au niveau du paysan. Les performances des ânes, des chevaux et des bœufs de trait ont été comparées lors d'essais sur les capacités de travail. Les équidés sont plus adaptés à un travail rapide de faible intensité où leur plus grande vitesse de travail est mise à profit. A des intensités de travail plus fortes, où la vitesse est moins importante, le surplus pondéral des bœufs devient plus avantageux. La récupération du rythme cardiaque après le travail constitue une bonne indication de la fatigue et de l'état physique des équidés dans des conditions normalisées d'expérimentation. Bien que les dépenses énergétiques liées au travail soient généralement faibles, la qualité du fourrage peut être si indigente que les animaux se trouvent dans l'incapacité d'en ingérer en quantité suffisante pour couvrir leurs besoins énergétiques lors de travaux et perdent ainsi du poids pendant la saison des travaux. Cependant, après une complémentation alimentaire pendant la saison sèche, des améliorations ne sont pas toujours observées dans les performances au travail et l'aspect économique doit être envisagé pour chaque cas. La disponibilité alimentaire, les maladies et le stress à la chaleur, principales contraintes agissant sur les performances des bœufs et des ânes de trait d'Afrique sub-saharienne, sont discutés.
\end{abstract}

\section{INTRODUCTION}

Les animaux de trait jouent un rôle important dans la production agricole et le transport en Afrique sub-saharienne. Si dans les grandes exploitations agricoles commerciales africaines l'utilisation de l'énergie animale a été remplacée par les tracteurs, la traction animale demeure une technologie adaptée et d'avenir pour les exploitations de plus petite taille, principalement pour des raisons économiques et agro-écologiques. Les coûts d'acquisition et d'entretien des tracteurs sont élevés dans beaucoup de pays subsahariens alors que l'énergie animale est moins chère, localement disponible et d'entretien facile par comparaison à la motorisation.

1. CTVM, University of Edinburgh, Easter Bush, Roslin, Midlothian, EH25 9RG, Scotland, United Kingdom

2. Cirad-emvt, BP 5035, 34032 Montpellier Cedex 1, France

* Cet article est publié conjointement en anglais dans Tropical animal health and production
Certaines surfaces cultivables, en particulier les flancs de montagne et les vallées escarpées, ne sont pas accessibles aux tracteurs et ne peuvent être travaillées que grâce à l'énergie animale ou à la main.

La majorité des paysans d'Afrique sub-saharienne pilotent des systèmes de production mixte sur de petites exploitations de dimension inférieure à 4 ha. Pour ces paysans, l'énergie animale offre une alternative à la culture manuelle tant pour les cultures vivrières (céréales dans les zones semi-arides, tubercules et plantains dans les zones humides) que pour les cultures de rente (riz, coton, arachide, café, banane et cacao). L'énergie animale a été introduite en Afrique sub-saharienne le siècle dernier et son utilisation n'a cessé de crôitre à ce jour (37). Cependant, le labour manuel prédomine toujours. Environ 80 p. 100 de l'énergie agricole utilisée pour le travail du sol est produite manuellement et l'énergie animale ne contribue que dans 15 p. 100 des surfaces cultivées en Afrique sub-saharienne $(15,20)$. Fall (12) indique qu'il est important de promouvoir l'utilisation de l'énergie animale en Afrique sub-saharienne où la production alimentaire est en déclin dans 
plusieurs pays, afin de couvrir une demande croissante en nourriture. L'énergie animale est particulièrement importante dans les milieux semi-arides où le respect du calendrier agricole est essentiel pour la réussite des opérations culturales (12). Les paysans ayant accès à l'énergie animale ont davantage tendance à étendre leurs surfaces cultivées que ceux utilisant les techniques manuelles de labour (16). Bien que cette pratique d'extension induit souvent une baisse des rendements à l'hectare, le revenu global de l'exploitation peut augmenter du fait de l'augmentation des surfaces cultivées.

L'adoption de l'énergie animale dans la production agricole est fréquemment associée à l'utilisation d'autres intrants tels que les semences sélectionnées et les engrais, si bien qu'il est souvent difficile de distinguer ce qui a produit les plus grands effets. Dans les régions cotonnières de l'Afrique de l'Ouest, par exemple, où l'énergie animale a été encouragée par les sociétés cotonnières, la production vivrière a augmenté dans certains cas avec l'introduction de l'énergie animale. La production vivrière a non seulement bénéficié d'un travail du sol plus approprié, mais aussi de l'utilisation des engrais appliqués pour la culture du coton (27).

L'extension des surfaces cultivées à l'aide de l'énergie animale est possible parce qu'elle fait gagner du temps aux paysans pour préparer et pour semer les champs, par rapport aux méthodes manuelles de préparation du sol. Le gain de travail peut être considérable. Par exemple, en comparant des rendements horaires à l'hectare du travail manuel et du travail utilisant l'énergie animale dans la production du riz et du maïs au Nigeria central, on obtient : $315 \mathrm{~h} / \mathrm{ha}$ pour le riz (labour et hersage) et $65,5 \mathrm{~h} / \mathrm{ha}$ pour le maïs (billonnage) en travail manuel, contre seulement $94 \mathrm{~h} / \mathrm{ha}$ pour le riz et $28 \mathrm{~h} /$ ha pour le maïs avec des bœufs de trait (24).

Cependant, quand les surfaces cultivées augmentent, l'utilisation de l'énergie animale ne se traduit pas par une réduction de la charge de travail totale sur l'exploitation. Le plus souvent, elle déplace les pointes de travail sur une autre période du calendrier des travaux. En labour manuel, le goulot d'étranglement est souvent la préparation du sol avant les semis. L'utilisation de l'énergie animale fait certes sauter ce goulot, mais il peut être transféré au niveau de la lutte contre les adventices, particulièrement lorsque les surfaces cultivées augmentent de façon notable. Les grandes exigences en travail dans la lutte contre les mauvaises herbes peuvent partiellement être satisfaites par l'utilisation de l'énergie animale pour sarcler entre les rangs. Cela n'est possible que si la culture a été semée en lignes et que si le matériel de sarclage mécanique est disponible sur l'exploitation. La réduction de la durée du travail de sarclage peut être considérable si les animaux sont utilisés entre les rangs avec un ensemble sarcleur mécanique. Par exemple, une étude réalisée sur ce sujet en Tanzanie (22) a montré que le temps de travail pour le sarclage du maïs est réduit jusqu'à 80 p. 100 par rapport au sarclage manuel. Cependant, si le sarclage dans les lignes est mauvais, les rendements à la récolte baissent (5 à $3,3 \mathrm{t} / \mathrm{ha}(22))$. Une combinaison du sarclage mécanique et du sarclage manuel à l'intérieur des lignes restaure les rendements à $5 \mathrm{t} /$ ha avec une réduction de travail de 40 p. 100 par rapport au sarclage intégralement manuel.

Pour réussir dans la production agricole, les paysans utilisant l'énergie animale doivent maîtriser les besoins de l'animal aussi bien que ceux de la plante. L'utilisation efficiente des animaux de trait dépend d'une bonne compréhension de leurs capacités de travail, de leurs besoins d'élevage et nutritionnels, ainsi que des facteurs pouvant influencer leurs performances. Tous ces aspects sont abordés dans cette synthèse dans le contexte de l'utilisation des animaux de trait dans les systèmes de production vivriers en Afrique sub-saharienne.

\section{- CAPACITES DE TRAVAIL DES DIFFEREN TES ESPECES AN IM ALES}

Le travail (en joules : J) est égal à la force (en newtons : N) multiplié par la distance (en mètres : $\mathrm{m}$ ) :

$$
\mathrm{W}(\mathrm{J})=\mathrm{F}(\mathrm{N}) \cdot \mathrm{d}(\mathrm{m})
$$

Quand un animal utilise de l'énergie pour tracter un outil ou pour porter une charge sur une certaine distance, il produit un travail. La quantité de travail de traction qu'un animal peut effectuer dépend du déplacement et de la force de traction générée. Pour un effort de traction donné, c'est la vitesse qui détermine la puissance de l'animal, c'est-à-dire la rapidité avec laquelle il effectue le travail. Un grand animal peut supporter une force de traction plus importante qu'un petit, mais quel que soit l'animal, quand la force de traction augmente, la vitesse diminue. Un animal labourant un vertisol lourd (force de traction importante) travaille plus lentement que lorsqu'il tire une charrette bien équilibrée sur une route goudronnée (force de traction faible). Les relations qui existent entre le poids vif, la force de traction, la distance parcourue et la vitesse ont été étudiées en station sur différents animaux de trait dans des essais de performances $(7,36,43)$. Ces études ont permis de faire des comparaisons entre les espèces. Par exemple, les forces de traction qui permettent d'obtenir un travail de traction maximal ont été déterminées pour différents animaux et combinaisons d'animaux par Betker et Kutzbach (7). Ils ont étudié les performances au travail d'ânes en attelage individuel au Niger (poids vif : 130-150 kg). Un intervalle de force $\left(\mathrm{F}_{90}\right)$ a été déterminé lorsque le travail atteint au moins 90 p. 100 du potentiel maximal $\left(\mathrm{W}_{\max }\right)$ (figure 1). Sur les ânes étudiés, les forces de traction pour lesquelles le travail atteint son maximum se situent entre 130 et 230 N. Quand la force optimale est rapportée au poids vif, elle atteint une moyenne de 12,9 p. $100 \mathrm{du}$ PV. Cette valeur est plus élevée que celles observées sur d'autres combinaisons d'animaux de trait, c'est-à-dire des paires de bœufs, de vaches et des monobœufs (moyenne 12 p. 100 du PV (7)). Le rapport entre le travail maximal et le poids vif était inférieur à celui des bovins montrant que si les ânes ont relativement une force de traction supérieure à celle des bovins, ils sont toutefois moins endurants qu'eux (7). Prasad et coll. (33) ont trouvé des résultats similaires sur des attelages à quatre ânes ou à quatre bœufs au Zimbabwe, mais les ânes étaient attelés par des jougs, ce qui a accéléré l'apparition de la fatigue chez ces animaux. Un attelage de quatre ânes (PV moyen $120 \mathrm{~kg}$ ) a travaillé à la même vitesse qu'un attelage de quatre bœufs (PV moyen $326 \mathrm{~kg}$ ) mais n'a effectué que $2 \mathrm{~h}$ de travail tandis que les bœufs ont poursuivi leur effort durant $4 \mathrm{~h}$. Dans une étude ultérieure (18), la vitesse de travail de quatre ânes

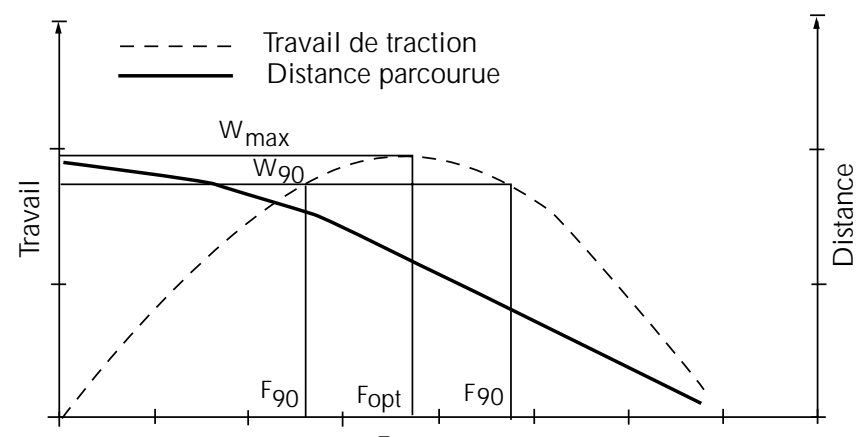

Force

Figure 1 : relations entre la force de traction, la distance parcourue et le travail de traction lorsque les animaux tractent un outil (Betker et Kutzbach, 1991). 
(PV moyen : $144 \mathrm{~kg}$ ) portant des bricoles thoraciques s'est avérée finalement plus faible que celle de quatre bœufs jougués (PV moyen : $367 \mathrm{~kg}$ ). L'augmentation du nombre d'animaux dans un attelage augmente évidemment sa capacité de traction mais pas suivant un terme constant. Quand le nombre d'animaux augmente, la contribution de chacun d'eux à l'effort de traction devient plus faible.

Au Cameroun, des essais de performance sur des ânes (PV : 100-150 kg), des chevaux (PV : 200-300 kg) et des zébus (PV : $300-450 \mathrm{~kg}$ ) ont été conduits pour déterminer les forces de traction permettant d'obtenir un travail optimal (43). Cette étude a permis d'établir des tables de vitesse, de durée de travail et de dépense énergétique liée au travail estimé, sur différents intervalles de force de traction et différents poids vifs pour chaque espèce. Le tableau I résume les variations de vitesse et de durée de travail quand on se situe à 95 p. 100 du travail de traction maximum pour les différentes espèces et les différents intervalles de poids vif (43). Les équidés ont tendance à travailler plus rapidement que les bovins. Quand la force de travail augmente, la vitesse et la durée de travail diminuent. Enfin, plus un animal est lourd, plus il montre de l'endurance lors de travaux de traction de fortes intensités.

Les essais de performance en milieu contrôlé permettent d'identifier l'espèce et le type d'animal (gabarit) les mieux appropriés pour effectuer un travail attelé déterminé. Cependant, la plupart des essais en milieu contrôlé sont menés sur des animaux bien dressés et élevés dans des conditions éloignées des conditions paysannes. Manifestement, des animaux moins expérimentés, mal nourris ou malades seront incapables de produire d'aussi bonnes performances. Des conditions de travail défavorables telles que la pression du climat (chaleur et humidité), les harnachements mal adaptés et des conditions de sol mauvaises peuvent toutes réduire le rythme de l'effort et donc affecter la quantité de travail effectuée par jour.

\section{MESU RES DES PERFO RMAN CES} SUR LE TERRAIN

En réalité, le travail produit n'est pas seulement fonction de l'animal, mais il est influencé par l'outil, la profondeur du travail, l'opérateur et les conditions environnementales et édaphiques. Le tableau II illustre, pour diverses tâches, les variations des performances des animaux de trait obtenues en milieu paysan dans les conditions de l'Afrique sub-saharienne. Les efforts de traction nécessités par les travaux de préparation du sol (labour, billonnage) peuvent atteindre $1000 \mathrm{~N}$, mais le plus souvent ils varient entre 600-900 N (tableau II). Ceci explique que les bœufs sont généralement les animaux de trait préférés pour le travail de préparation du sol, en particulier pour les sols lourds. Si les ânes doivent être utilisés pour un travail identique, un attelage d'au moins quatre ânes est nécessaire pour obtenir un bon rendement de travail et la possibilité de cultiver une surface raisonnable par jour. Les forces de traction requises par les semis et les sarclages mécaniques sont plus faibles que celles du travail de préparation du sol (souvent pas plus de $270 \mathrm{~N}$ pour un semis sur sols sableux (5)). Semis et sarclages peuvent donc être exécutés par un seul bœuf, un seul âne ou un seul cheval. L'avantage des chevaux et des ânes réside dans leurs déplacements plus rapides dans l'ensemble par rapport aux bœufs. De ce fait, lorsque les forces de traction requises sont faibles (cas du transport, du semis et du sarclage sur sols légers sablonneux), les équidés sont sélectionnés comme animaux de traction. Par exemple, en Afrique de l'Ouest (Sénégal, Mali et Niger), le semis direct de l'arachide, du mil et du sorgho à l'aide d'un seul âne ou d'un petit cheval est une pratique très répandue pour permettre de semer rapidement de grandes surfaces en vue d'augmenter les chances de réussite de la culture, sur les sols légers sableux des zones semi-arides où les pluies sont aléatoires.

Tableau I

Vitesse moyenne, puissance et durée du travail attelé d'ânes, de chevaux et de zébus ( $n=2$ ) tractant un traîneau à différentes intensités de force de traction,

lors d'essais de performances (séances d'essai) au Cameroun (Vall, 1996)

\begin{tabular}{|c|c|c|c|c|c|c|}
\hline Espèces & $\begin{array}{l}\text { Poids vif } \\
\text { (kg) }\end{array}$ & $\begin{array}{c}\text { Force } \\
\text { de traction (N) }\end{array}$ & $\begin{array}{c}\text { Force } \\
\text { de traction } \\
\text { (kg \% poids vif) }\end{array}$ & $\begin{array}{c}\text { Vitesse } \\
(\mathrm{m} / \mathrm{s})\end{array}$ & $\begin{array}{l}\text { Durée } \\
\text { (h:min) }\end{array}$ & $\begin{array}{c}\text { Puissance } \\
\text { (W) }\end{array}$ \\
\hline Anes & $\begin{array}{l}100 \\
100 \\
125 \\
125 \\
150 \\
150\end{array}$ & $\begin{array}{r}98 \\
147 \\
147 \\
196 \\
147 \\
245\end{array}$ & $\begin{array}{l}10 \\
15 \\
12 \\
16 \\
10 \\
17\end{array}$ & $\begin{array}{l}0,88 \\
0,76 \\
0,83 \\
0,73 \\
0,88 \\
0,72\end{array}$ & $\begin{array}{l}5: 46 \\
3: 43 \\
4: 53 \\
3: 22 \\
5: 46 \\
3: 08\end{array}$ & $\begin{array}{r}86 \\
112 \\
122 \\
143 \\
129 \\
176\end{array}$ \\
\hline Chevaux & $\begin{array}{l}225 \\
225 \\
250 \\
250 \\
275 \\
275 \\
300 \\
300\end{array}$ & $\begin{array}{l}245 \\
343 \\
294 \\
392 \\
294 \\
392 \\
343 \\
441\end{array}$ & $\begin{array}{l}11 \\
16 \\
12 \\
16 \\
11 \\
15 \\
12 \\
15\end{array}$ & $\begin{array}{l}0,93 \\
0,90 \\
0,92 \\
0,90 \\
0,93 \\
0,91 \\
0,93 \\
0,90\end{array}$ & $\begin{array}{l}6: 22 \\
4: 24 \\
5: 57 \\
4: 13 \\
6: 28 \\
4: 49 \\
6: 06 \\
4: 38\end{array}$ & $\begin{array}{l}228 \\
309 \\
270 \\
353 \\
273 \\
357 \\
319 \\
397\end{array}$ \\
\hline Bovins & $\begin{array}{l}350 \\
350 \\
400 \\
400 \\
450 \\
450\end{array}$ & $\begin{array}{l}343 \\
540 \\
392 \\
589 \\
392 \\
638\end{array}$ & $\begin{array}{r}10 \\
16 \\
10 \\
15 \\
9 \\
15\end{array}$ & $\begin{array}{l}0,78 \\
0,67 \\
0,78 \\
0,68 \\
0,81 \\
0,67\end{array}$ & $\begin{array}{l}6: 24 \\
4: 17 \\
6: 24 \\
4: 38 \\
6: 39 \\
4: 22\end{array}$ & $\begin{array}{l}268 \\
362 \\
306 \\
401 \\
318 \\
427\end{array}$ \\
\hline
\end{tabular}



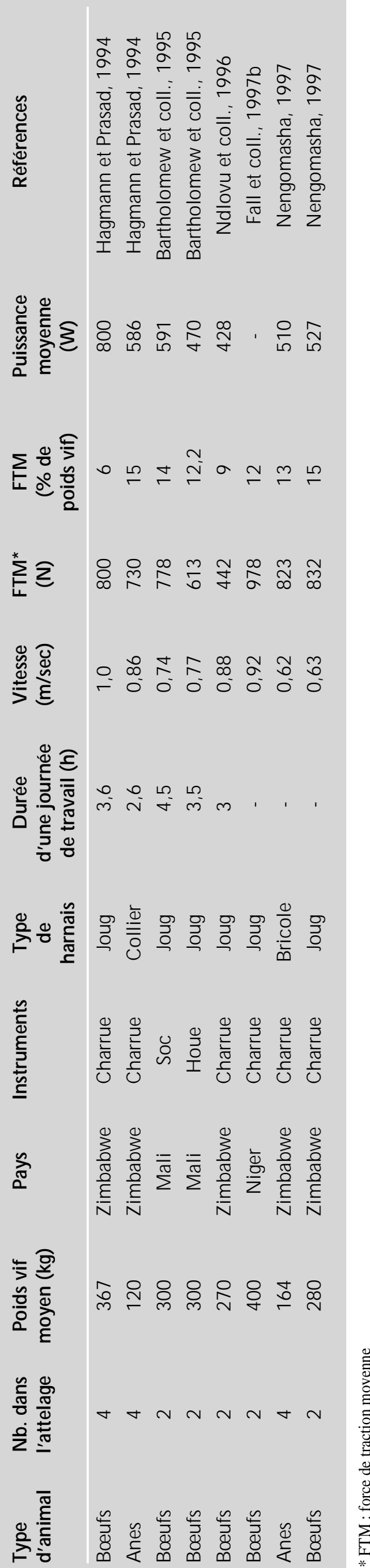

\section{- ADAPTATION ET ESTIMATION DE LA FATIGUE DES ANIMAUX AU TRAVAIL}

Certains chercheurs ont comparé les performances des animaux de trait sur des périodes successives. Par exemple Fall et coll. (13) et Bartholomew et coll. (4) ont trouvé des vitesses et des rendements de travail journaliers plus importants la seconde semaine de travail, par rapport à la première, du fait d'une adaptation plus poussée des animaux à l'effort retrouvée après la période creuse de la saison sèche. Cette adaptation s'opère malgré les pertes de poids observées pendant cette même période. D'autres tentatives pour déterminer objectivement les effets du travail sur l'animal ont été testées. Upadhyay et Madan (41) ont élaboré un système d'appréciation de la fatigue basé sur l'observation de huit paramètres comportant des critères qualitatifs et quantitatifs. Chaque paramètre est estimé et noté sur 5 et leur somme permet d'attribuer une note totale. Une technique largement acceptée pour apprécier l'adaptation de l'animal à l'effort est la récupération cardiaque après un exercice donné. Cette technique est couramment utilisée chez les athlètes et les chevaux de course. Au Cameroun, elle a été appliquée sur des chevaux et des ânes au travail (43). La fréquence cardiaque chute d'abord rapidement pendant la première minute de repos après l'arrêt du travail, puis décrôit plus lentement. La mesure de la fréquence cardiaque trois minutes après le travail s'est avérée un bon indicateur de la récupération cardiaque chez les chevaux et les ânes. Vall (43) propose comme guide approximatif de la bonne récupération des équidés les valeurs suivantes de la fréquence cardiaque maximale trois minutes après le travail : 80 battements/minute dans le cas d'un travail léger d'intensité inférieure à 15 p. $100 \mathrm{PV}$, et 90 battements/minute dans le cas d'un travail lourd d'intensité supérieure à 15 p. 100 PV (figure 2). Cependant, les variations individuelles de la récupération cardiaque peuvent être considérables et la fréquence cardiaque peut aussi être affectée par le type de travail entrepris (42). Avec le temps, la technique semble être plus utile pour contrôler l'adaptation et le confort individuels des animaux avec une charge de travail normalisée dans chaque essai.

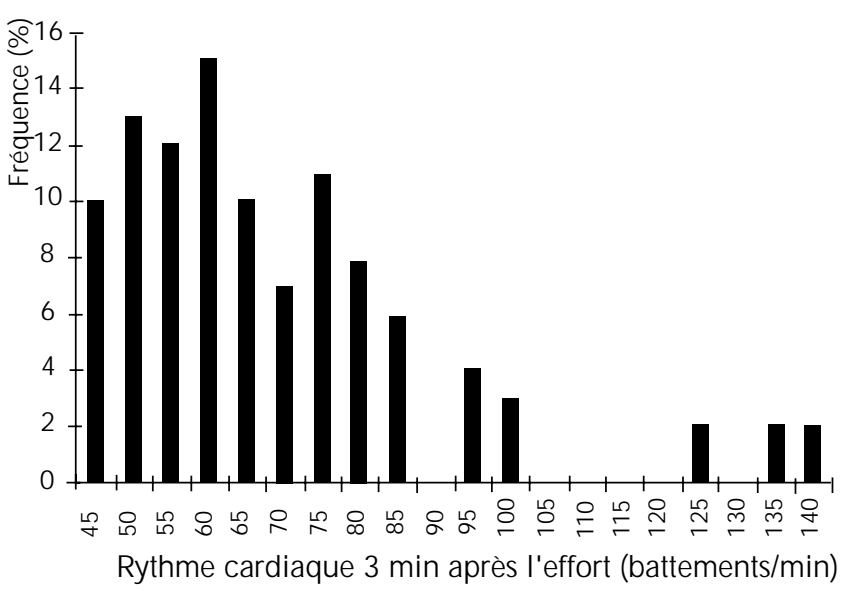

Figure 2 : distribution de la fréquence cardiaque 3 min après l'arrêt de l'effort (en battements par minute) enregistrée sur des chevaux au Cameroun à la suite de séances d'essai menées à une intensité de l'effort équivalente à 10-15 p. 100 PV (Vall, 1996). 


\section{-DEPEN SE EN ERGETIQUE LIEE AU TRAVAIL}

La dépense énergétique nette liée au travail est déterminée par la distance parcourue, la charge déplacée et le type de sol sur lequel l'animal évolue. La distance parcourue pendant le travail est influencée par l'opérateur, la tâche entreprise, la durée du travail, les conditions climatiques et la santé de l'animal. Un animal travaillant intensément sur une courte période peut dépenser moins d'énergie que lorsqu'il travaille plus légèrement sur une longue période. Par exemple, un animal attelé à une charrette chargée peut dépenser plus d'énergie qu'un animal effectuant un labour, bien que le labour nécessite une force de traction supérieure, en raison de la plus grande distance parcourue lors du transport. Les dépenses énergétiques liées à la marche, à la traction et au port des charges ont été déterminées pour la plupart des animaux de trait $(10,25,44)$. Les dépenses énergétiques de traction et de portage ne varient pas avec la surface sur laquelle les animaux marchent $(11,14)$, à la différence de la dépense énergétique liée à la marche. Cette dernière peut être multipliée par quatre entre un sol dur et un sol meuble gorgé d'eau (11). Lors d'un labour, l'animal marchant dans la raie peut dépenser jusqu'à 20 p. 100 d'énergie de plus que celui marchant sur le guéret (11). Tous ces facteurs doivent être pris en compte lors de l'estimation de la dépense énergétique journalière d'un animal au travail.

Les dépenses énergétiques journalières liées au travail varient selon les conditions de travail et selon l'animal mais, exprimées comme un multiple des dépenses d'entretien, elles restent relativement faible. Même dans de bonnes conditions d'alimentation et de conduite, les bovins de trait dépensent rarement plus de 1,8 fois la valeur de l'entretien au cours d'une journée de travail (23). Le cheval de trait de grand format est l'animal de trait qui dépense le plus d'énergie pour effectuer un travail. Son gabarit élevé lui confère une capacité de traction importante. Il peut travailler des heures sur une longue période. En contrepartie, l'augmentation de sa dépense énergétique par rapport à l'entretien est élevée : 2,242,5 fois l'entretien (32). Les ânes semblent travailler plus efficacement seulement pendant 2-4 h d'affilée. Leur journée de travail peut être augmentée à 4-6 h en les faisant travailler le matin puis l'après-midi. Cette pratique est souvent utilisée par les paysans du Zimbabwe qui, en vue d'optimiser leurs rendements, cherchent à respecter au mieux le calendrier cultural (18).

\section{SO U RCES D'ENERGIE POUR LE TRAVAIL}

En Afrique sub-saharienne, le mil, le sorgho, le maïs, le riz, l'arachide et le niébé sont les principales cultures de la saison des pluies et dépendent de la quantité de pluie reçue. Les résidus de ces cultures constituent une partie importante du fourrage disponible pour les animaux de trait. Les pâturage naturels et les talus des routes représentent une autre ressource fourragère pour les animaux de trait. La qualité nutritionnelle de tous ces aliments est souvent très basse (40), particulièrement en fin de saison sèche et au début de la saison des pluies lorsque les animaux effectuent la plupart des travaux. Le travail réduit le temps consacré à la consommation d'aliments tout en augmentant les besoins énergétiques des animaux. Dès lors, l'ingestion de ces aliments de base par les animaux de trait le jour du travail reste insuffisante pour couvrir leurs besoins alimentaire $(29,31)$. Les animaux de trait perdent généralement du poids pendant la saison des travaux, sauf en cas d'apport d'un complément alimentaire suffisamment riche et facilement assimilable $(5,7)$.

L'aptitude des animaux de trait à consommer et à digérer les aliments disponibles pendant la saison des travaux est un élément important dès lors qu'elle permet de minimiser les pertes de poids durant cette période. Les ânes apparaissent plus aptes que les autres équidés à digérer la fraction fibreuse des rations de faible valeur nutritive et maintiennent une consommation plus élevée que les bovins pour des rations similaires ( 9 ; Smith, comm. pers.). Ceci leur confère un avantage sur les autres animaux de trait lorsqu'il s'agit de couvrir les besoins énergétiques quotidiens avec des rations pauvres.

\section{- CO M PLEM EN TATIO N DES ANIM AU X DE TRAIT}

Au Zimbabwe, la complémentation de la ration de base des bœufs de trait Mashona en saison sèche (constituée de pâturage naturel et de tiges de maïs) avec de la paille d'arachide, à raison de $400 \mathrm{~g} / \mathrm{animal} / \mathrm{j}$ pendant 70 jours ou $800 \mathrm{~g} / \mathrm{animal} / \mathrm{j}$ pendant 35 jours avant le labour (novembre), a conduit à une augmentation significative du poids vif des animaux (respectivement 301 et $312 \mathrm{~kg}$ avant le labour) par rapport aux bœufs non complémentés $(276 \mathrm{~kg})$. Tous les animaux ont perdu du poids pendant le labour mais le travail journalier effectué, la puissance et la surface labourée ont été plus élevés chez les animaux complémentés. La durée courte ou longue de la complémentation avant le labour n'a pas eu d'impact sur l'amélioration significative des performances (17). Bartholomew et coll. (4) ont aussi montré que la capacité de travail est plus élevée chez des animaux lourds que chez des animaux légers, et ce malgré l'amaigrissement durant le travail.

Plus le gabarit d'un animal est important, plus l'animal est apte à produire un travail de traction et moins il en subit le stress. Bien qu'il ait été souvent suggéré que les animaux de trait doivent être en bonne condition au début de la saison des travaux, il a rarement été démontré que les animaux de trait en bon état corporel en début de saison travaillent plus vite et/ou plus longtemps que ceux en moins bon état. Des études réalisées au Mali $(4,5)$ et au Niger (13) ont indiqué que c'est le poids vif et non l'état corporel qui détermine principalement la capacité au travail des bœufs de trait. $\mathrm{Au}$ Niger, pour des animaux de même poids vif $(360 \mathrm{~kg}$ ), les maigres (note d'état corporel de 3 sur une échelle de 9 (30)) ont effectué un travail quotidien identique ou supérieur (8 $262 \mathrm{~kJ})$ à des animaux en meilleur état corporel (note 4 à 6 , force 7437 $7876 \mathrm{~kJ}$ ). Si l'on ne tient pas compte de l'état corporel, ce sont les animaux les plus lourds qui fournissent le plus de travail de traction (13). Teleni (38), dans une étude sur les buffles en Asie, indique que la force de traction maximale supportée des animaux au travail est égale à $12-15$ p. $100 \mathrm{PV}$. Il suggère que la perte de poids durant la saison des travaux n'est pas un problème dans la mesure où cette baisse ne dépasse pas 12 à 15 p. 100 du poids vif de l'animal (38). Si la baisse de poids n'atteint pas un tel niveau, la complémentation des animaux de trait peut ne pas être efficace (17).

Dans des villages maliens, la valeur nutritive de la paille de brousse de saison sèche a été améliorée par addition d'urée à 4 p. 100. En comparaison avec un groupe de bovins témoins (PV : 280-330 kg), les animaux consommant de la paille traitée ont perdu relativement moins de poids pendant la saison des travaux (respectivement -200 contre -100 g/animal/j). Les bœufs qui ont reçu la paille traitée et un complément additionnel de $1 \mathrm{~kg} / \mathrm{j}$ d'aliment à forte proportion de tourteau de coton ont enregistré des gains de poids de $200 \mathrm{~g} / \mathrm{animal} / \mathrm{j}$ entre avril et juin (21). Cependant, la complémentation de saison sèche n'a eu aucun effet sur le rendement journalier au travail des animaux pendant la saison des cultures suivante. 
En définitive, une étude économique de la complémentation de saison sèche doit être considérée dans chaque cas. Dans certaines situations, par exemple en cas de saison des cultures courte, la complémentation n'est pas économiquement justifiable. $\mathrm{Si}$, en revanche, la saison des cultures est longue, le transport attelé en saison sèche actif ou bien si la pratique de l'embouche existe, les retombées économiques de la complémentation peuvent valoir la peine.

Les concentrés alimentaires constituent une complémentation appropriée de la ration de base des animaux au travail, du fait de leur haut niveau énergétique et de la facilité de leur distribution avant et après le travail. Cependant, les aliments concentrés ne sont pas toujours disponibles et sont souvent trop chers pour les paysans. Les fanes de légumineuses (arachide, niébé, dolique, etc.) sont des fourrages de qualité permettant de réduire la carence de nourriture pendant la saison sèche et constituent des rations d'appoint pendant les travaux $(2,17)$. D'autres aliments tels les sous-produits agro-industriels (tourteau, mélasse), les feuilles d'arbres (ficus), les coques (légumineuses, coton) disponibles localement peuvent également servir d'alternatives pour la complémentation des animaux de trait.

\section{- CONTRAINTES DE L'EN VIRO N N EM ENT SU R LES PERFO RM ANCES}

En Afrique sub-saharienne, la majorité des animaux de trait vivent dans les zones agro-écologiques semi-arides, soudaniennes et sahéliennes où la saison sèche dépasse souvent cinq mois. Dans ces zones, l'approvisionnement en aliments est le problème le plus important rencontré par les propriétaires d'animaux de trait. L'élevage d'animaux de trait dans les zones d'Afrique plus humides subit les contraintes du contexte pathologique, en particulier la trypanosomose. L'usage des bovins devient possible au fur et à mesure de l'éradication des glossines dans une région et les paysans se convertissent peu à peu à la traction animale. Au cours des 20 dernières années, une faible augmentation des bœufs de trait a été enregistrée en Afrique de l'Est et du Sud. Quant à l'Afrique de l'Ouest et l'Afrique centrale, l'extension des bœufs de trait progresse davantage dans le Sud, dans les régions sub-humides où l'infestation des glossines est en recul (37). En Afrique de l'Ouest, les taurins trypanotolérants, bien que de petite taille, ont constitué une importante source d'énergie dans les régions où les trypanosomoses animales sont endémiques.

En Afrique sub-saharienne, la chaleur est une autre contrainte qui agit sur les performances des animaux de trait. A la différence des animaux de rente qui peuvent s'abriter à l'ombre quand la température augmente, les animaux de trait doivent habituellement travailler au soleil, souvent à des températures très supérieures à $20{ }^{\circ} \mathrm{C}$.

Les effets de la chaleur et des pathologies sur les performances des animaux de trait ont été étudiés pour améliorer la conduite des animaux de trait dans les milieux où ces facteurs sont problématiques.

\section{Stress thermique}

Le métabolisme énergétique lié à la contraction musculaire produit une quantité d'extra-chaleur importante. Afin de maintenir l'homéothermie et de poursuivre le travail, l'animal de trait dissipe l'extra-chaleur par différents mécanismes thermolitiques. En climat chaud, la dissipation de chaleur par convection n'est pas suffisante comme cela peut être le cas en climat tempéré. L'animal dissipe activement la chaleur produite par voie évaporatoire grâce à la sudation, au halètement et à l'émission de bave. En climat chaud et humide, l'efficacité avec laquelle l'animal dissipe l'extrachaleur liée au travail conditionne la quantité de travail produite.

Certains animaux sont plus aptes que d'autres à produire un travail en climat chaud. Plusieurs pays africains ont encouragé et entrepris le croisement des races locales avec des animaux exotiques des zones tempérées en vue d'améliorer le potentiel génétique de la production laitière et des animaux d'élevage. Ces programmes ont permis de produire de nombreux métisses disponibles pour le travail. L'avantage des métisses, qui peuvent fournir une puissance de travail plus importante grâce à une plus grande taille, est contrebalancé par une moins bonne aptitude à supporter la chaleur. Là où les animaux travaillent de longues heures à des températures et une humidité élevées, il est finalement plus avantageux et plus sûr de travailler avec des animaux locaux de plus petite taille mais possédant une plus grande tolérance à la chaleur que les métisses (39).

$\mathrm{Au}$ Cameroun, les variations de la température rectale (TR) des zébus, des ânes et des chevaux de trait au repos ont été étudiées en fonction du temperature humidity index (THI*) (43). Les corrélations suivantes ont été établies :

$$
\begin{aligned}
& \text { - zébu }: \text { TR }=29,75+0,11 . \text { THI }\left(\operatorname{ddl} 342 ; \mathrm{R}^{2}=0,56\right) \\
& \text { - cheval }: \text { TR }=31,50+0,08 . \text { THI }\left(\operatorname{ddl} 352 ; \mathrm{R}^{2}=0,33\right) \\
& \text { - âne }: \text { TR }=26,56+0,14 . \text { THI }\left(\operatorname{ddl} 362 ; \mathrm{R}^{2}=0,56\right)
\end{aligned}
$$

Les chevaux ont montré la plus faible réponse au THI et les ânes les plus fortes fluctuations de la température rectale. Les mêmes différences sont également observées au cours du travail. L'augmentation de la température rectale liée au travail a été plus importante pour les intensités de travail les plus élevées pendant la saison des pluies quand l'humidité et THI sont les plus élevés. Cette augmentation a été de $1-2{ }^{\circ} \mathrm{C}$ chez les ânes, de $0,5-1,8{ }^{\circ} \mathrm{C}$ chez les zébus et de $0,5-1,2{ }^{\circ} \mathrm{C}$ chez les chevaux.

Fall (12), au Niger, a étudié l'aptitude des bœufs à dissiper la chaleur liée au travail à différents gradients de température (17-35 $\left.{ }^{\circ} \mathrm{C}\right)$. Il a mesuré la température rectale, la fréquence respiratoire et l'augmentation maximale de température rectale $\left(\mathrm{TR}_{\mathrm{w}, \max }\right)$ des bœufs pendant le travail. Dans tous les cas, la température rectale et la fréquence respiratoire ont augmenté avec le travail. La puissance développée est corrélée négativement avec la température rectale et THI (figure 3). Il existe cependant une variation individuelle marquée de la capacité des bœufs à faire face à la chaleur. Les animaux gras ont plus de difficultés que les maigres pour maintenir leur homéothermie (12). Les estimations de la répétabilité de la température rectale avant le travail $(0,19)$, pendant le travail $(0,16)$, et des $\operatorname{TR}_{\mathrm{w} \text {,max }}(0,29)$ ont été généralement faibles. Fall (12) indique que pour les bœufs au travail $\mathrm{TR}_{\mathrm{w}, \max }$ serait un indice physiologique de thermotolérance plus approprié que les paramètres de fréquence respiratoire et de température rectale au repos identifiés par Buvanendran et coll. (8).

En définitive, il existe des différences dans l'aptitude des animaux à tolérer la chaleur. Les effets néfastes de la chaleur peuvent cependant être minimisés en travaillant pendant les moments les plus frais de la journée, avec des repos fréquents à l'ombre les jours de grande chaleur. Les animaux de trait doivent également avoir un accès illimité à l'eau de boisson une fois le travail achevé afin de

* THI $=0,72 .(\mathrm{Ts}+\mathrm{Th})+40,6 ;$ Ts : température sèche $; \mathrm{Th}:$ température humide en degrés Celsius. 
leur permettre de compenser les pertes d'eau par dissipations diverses. Rose (34) a montré par exemple que des chevaux accomplissant un exercice d'endurance sur plus de $100 \mathrm{~km}$ peuvent perdre par sudation 30 à 401 d'eau (5 à 7 p. 100 du PV). Les pertes d'eau des bœufs sont en général plus faibles dans les mêmes conditions de travail que les chevaux (A. Fall, comm. pers.).

\section{Maladie}

Au regard de l'investissement que constituent les animaux de trait pour les paysans et de leur dépendance vis-à-vis d'eux en période de culture, la prévention et le contrôle des plus graves maladies, en particulier pendant la saison des cultures, sont économiquement justifiables. Les maladies insidieuses sont plus difficiles à traiter. Elles peuvent ne pas tuer directement l'animal mais affecter gravement sa productivité et sa longévité. Il est certain que ces maladies
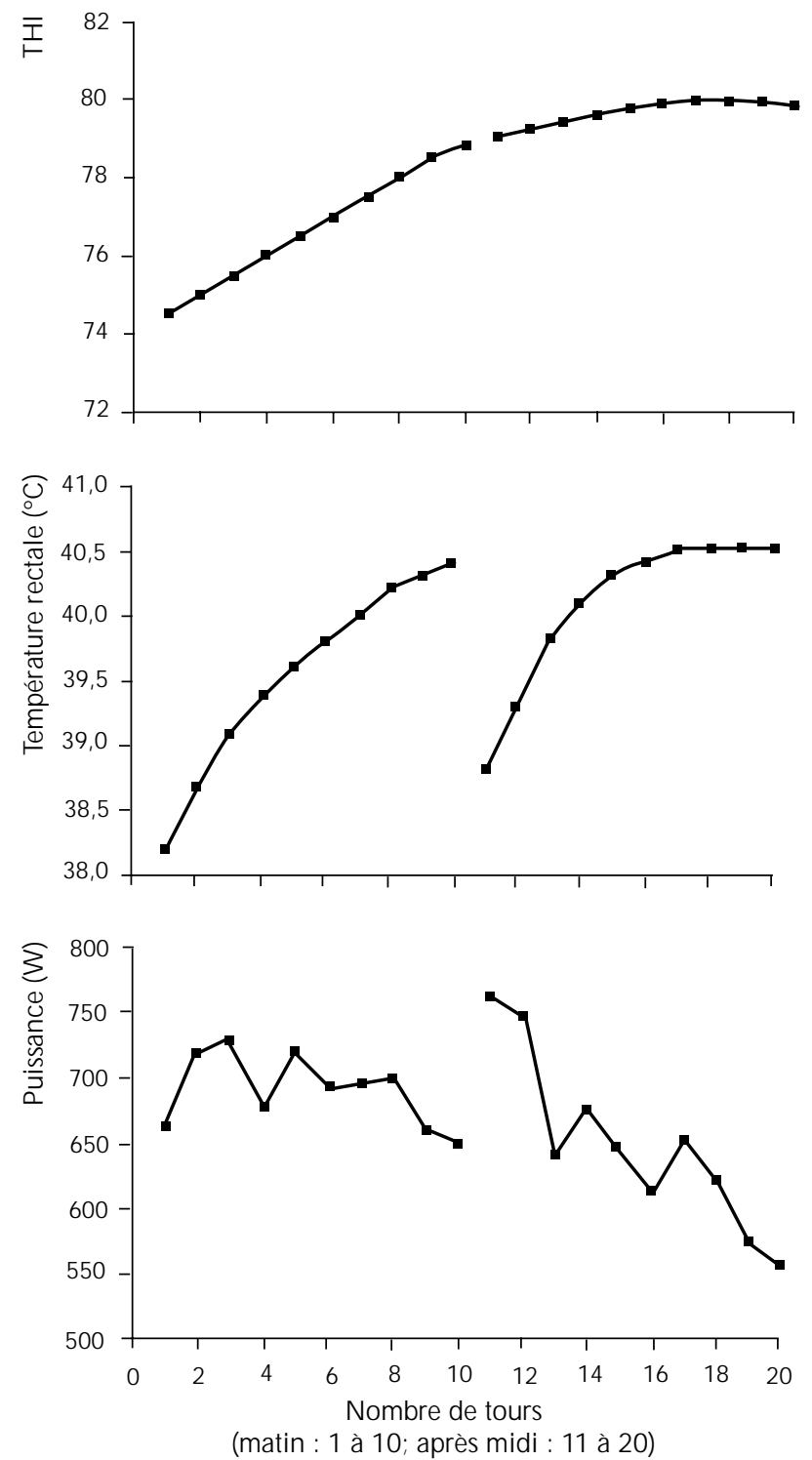

Figure 3 : variations moyennes du temperature humidity index (THI), de la température rectale et de la puissance de bovins travaillant pendant $4 \mathrm{~h}$ (10 tours de piste), le matin (tours 1 à 10) et l'après-midi (tours 11 à 20). La température rectale est enregistrée après chaque tour de piste. Les valeurs indiquées sont des moyennes calculées sur 18 animaux qui travaillent $4 \mathrm{~h} / \mathrm{j}$, à raison de $4 \mathrm{j} /$ semaine et durant 7 semaines (Fall, 1995). réduisent les rendements au travail et, conjuguées au stress du travail, prédisposent les animaux à d'autres maladies $(19,26)$. Le traitement de ces maladies peut ainsi être économiquement judicieux. En 1990 en Zambie, la dermatophilose a entraîné une perte par bovin estimée à 193 \$US par animal malade en termes de pertes de surfaces labourées et de revenus issus de la location de l'animal (35).

Les interactions entre le travail, la trypanosomose et l'alimentation ont été étudiées en Afrique de l'Ouest. La résistance naturelle des bovins trypanotolérants N'dama a été étudiée sur des animaux au travail et au repos. Une exposition primaire à Trypanosoma congolense a abouti à une baisse de l'hématocrite. L'anémie a été plus sévère chez les animaux qui travaillaient et elle n'a pas été améliorée par une complémentation de la ration de base par des fanes d'arachide de meilleure qualité (6). Il semblerait que si une bonne alimentation permet de mieux lutter contre la trypanosomose lorsque les animaux ne travaillent pas (1), dès lors que les animaux sont mis au travail la chimiothérapie devient nécessaire.

\section{CONCLUSION}

Au cours des deux dernières décennies en Afrique sub-saharienne, de nombreux travaux de recherche ont été entrepris sur les performances, les aptitudes et les besoins des animaux de trait. On peut raisonnablement affirmer que dans cette région du monde, les bœufs, les ânes et les chevaux de trait continueront d'être, en cette fin de $\mathrm{XX}^{\mathrm{e}}$ siècle, une solution d'avenir pour les petits exploitants pratiquant un système mixte ; avec une prédominance des bovins dans les zones où l'effort de traction requis est important et une préférence pour les équidés là où les sols sont plus légers et les efforts sont moins importants. La recherche a montré que les bœufs, les ânes et les chevaux peuvent apporter une contribution significative dans l'amélioration de la productivité des exploitations agricoles d'Afrique sub-saharienne et réduire la pénibilité du travail. Les ressources alimentaires et les divers intrants utiles pour maintenir ces animaux dans des conditions nutritionnelles appropriées et en bonne santé font souvent défaut. Avec un choix adéquat des animaux pour satisfaire les besoins en travail, une utilisation judicieuse des aliments disponibles et des pratiques d'exploitation permettant de minimiser les stress du travail sur l'animal, le petit exploitant agricole de l'Afrique sub-saharienne peut utiliser efficacement les animaux de trait pour satisfaire son besoin d'énergie mécanique pour les cultures et le transport.

\section{BIBLIO GRAPHIE}

1. AGYEMANG K., DWINGER R.H., TOURAY B.N., JEANNIN P. FOFANA D., GRIEVE A.S., 1990. Effects of nutrition on the degree of anaemia and liveweight changes in N'Dama cattle infected with trypanosomes. Livest. Prod. Sci., 26: 39-51.

2. ALFORD R.J., 1994. Working and feeding strategies of draught $\mathrm{N}$ 'Dama oxen in a Gambian village. MSc thesis, CTVM, University of Edinburgh, UK.

3. BARTHOLOMEW P.W., KHIBE T., LITTLE D.A., 1994. Effect of liveweight and body condition on work output from draft oxen. Trop. Anim. Health Prod., 26: 58-64.

4. BARTHO LOMEW P.W., KHIBE T., LITTLE D.A., BA S., 1993. Effect of change in body weight and condition during the dry season on capacity for work of draught oxen. Trop. Anim. Health Prod., 25: 50-58.

5. BARTHOLOMEW P.W., KHIBE T., LY R., 1995. In-village studies of the use of work oxen in Central Mali. Trop. Anim. Health Prod., 27: 241-248. 
6. BENNISON 」, CLEMENCE R.G., ARCHIBALD R.F., HENDY C.R.C., DEM PFLE L., 1998. The effects of work and two planes of nutrition on trypanotolerant draught cattle infected with Trypanosoma congolense. Anim. Sci., 66: 595-605.

7. BETKER J., KUTZBACH H.-D., 1991. Role of donkeys in agricultural mechanisation in Niger - potential and limitations. In: D. Fieldin, R.A Pearson eds., Donkeys, mules and horses in tropical agricultural development. Edinburgh, UK, U niversity of Edinburgh, p. 223-230.

8. BUVANENDRAN V., ADAMU A.M., ABUBAKAR B.Y., 1991. Heat tolerance of zebu and Friesian-zebu crosses in the Guinea savannah zone of Nigeria. Trop. Agric., Trinidad, 69: 394-396.

9. CUDDEFORD D., PEARSON R.A., ARCHIBALD R.F., MUIRHEAD R.H., 1995. Digestibility and gastro-intestinal transit time of diets containing different proportions of alfalfa and oat straw given to thoroughbreds, Shetland ponies, Highland ponies and donkeys. Anim. Sci., 62: 407-417.

10. DIJKMAN J.T., 1992. A note on the influence of negative gradients on the energy expenditure of donkeys walking, carrying and pulling loads. Anim. Prod., 54: 153-156.

11. DIJKMAN J.T., LAWRENCE P.R., 1997. The energy expenditure of cattle and buffaloes walking and working in different soil conditions. J. Agric. Sci., Cambridge, 128: 95-103.

12. FALL A., 1995. Factors affecting feed intake, energy expenditure and work output of oxen and bulls used for draught purposes in semi-arid West Africa. PhD thesis, U niversity of Edinburgh, UK.

13. FALL A., PEARSO N R.A., FERNAN DEZ-RIVERA S., 1997a. Nutrition of draught animals in semi-arid West Africa III. Effect of body condition prior to work and weight losses during work on food intake and work output. Anim. Sci., 64: 227-232.

14. FALL A., PEARSO N R.A., LAWRENCE P.R., 1997b. Nutrition of draught animals in semi-arid West Africa. I. Energy expenditure by oxen working on soils of different consistencies. Anim. Sci., 64: 209-215.

15. FAO, 1987. African agriculture: the next 25 years. Rome, Italy, FAO. 16. FRANCIS P.A., 1988. Ox draught power and agricultural formation in N orthern Zambia. Agric. Sys., 27: 15-28.

17. FRANCIS J., NDLOVU L.R., 1995. Improving feeding management and work performance of Mashona oxen through strategic supplementation with cobsheath-groundnut stover. Trop. Anim. Health Prod., 27: 249-257.

18. HAGMANN J., PRASAD V.L., 1994. The use of donkeys and their draught performance in smallholder farming in Zimbabwe. Conservation tillage for sustainable crop production systems. Harare, Zimbabwe, GTZ/AGRITEX/IAE. (Project research report 11)

19. HOFFMANN D., DALGLIESH R.J., 1985. A multi-disciplinary approach to health and disease in draught ruminants. In: J.W. Copland ed., Draught animal power for production. Canberra, Australia, ACIAR, p. 134-139. (Proc. series N o. 10)

20. JAHNKE H.E., SIEVERS M., 1981. Agricultural mechanisation and the demand for agricultural machinery and equipment in Africa to the year 2000. Vienna, Austria, UNID O / Rome, Italy, FAO. (Mimeograph)

21. KHIBET., BARTH O LOMEW P.W., 1993. Recherches aux possibilités de conditionnement des bœufs de labour à base de paille de brousse enrichie à l'urée et évaluation de son impact sur leur capacité de travail. In: P.R. Lawrence, K. Lawrence, J.T. Dijkman, P.H. Starkey eds. Research for development of animal traction in West Africa. Proc. 4th Work. West Africa Animal Traction Network, Kano, Nigeria, July 9-13, 1990. Addis Ababa, Ethiopia, ILCA, p. 175-179.

22. KWILIGWA E.M., SHETTO R.M., REES D.J., 1994. The use of animal-drawn cultivators for maize production in the southern highlands of Tanzania. In: P. Starkey, E. M wenya, J. Stares eds., Improving animal traction technology, Proc. 1st W ork. ATN ESA, Lusaka, Zambia, January 18-23, 1992. Wageningen, The Netherlands, CTA, p. 182-190.

23. LAWREN CE P.R., 1985. A review of the nutrient requirements of draught oxen. In: J.W. Copland ed., Draught animal power for production. Canberra, Australia, ACIAR, p. 59-68. (Proc. series N o. 10)

24. LAWREN CE P.R., DIJKMAN J.T., JANSEN H.G.P., 1997. The introduction of animal traction into inland valley regions. I. Manual labour and animal traction in the cultivation of rice and maize: a comparison. J. Agric. Sci., Cambridge, 129: 65-70.

25. LAWRENCE P.R., STIBBARDS R.J., 1990. The energy cost of walking, carrying and pulling loads on flat surfaces by Brahman cattle and swamp buffalo. Anim. Prod., 50: 29-39.

26. LEFEVRE P.-C., LHOSTE P., 1993. Diseases of draught animals in West Africa, In: D. O'N eill, G. Hendriksen eds., human and draught animal power in crop production. Proc. Silsoe Research Institute/CEC/FAO Work., Harare, Zimbabwe, January 18-22, 1993. Rome, Italy, FAO.

27. MAHDAVI G., 1992. Development of animal traction in cotton areas of French speaking African countries. In: G. den Hertog, J.A. van Huis eds., The role of draught animal technology in rural development. Proc. int. Semin., Edinburgh, UK, A pril 2-12, 1990. Wageningen, The N etherlands, Pudoc Scientific Publishers, p. 83-87.

28. NDLOVU L.R., FRANCIS J., HOVE E., 1996. Performance of draught cattle in communal farming areas in Zimbabwe after dry season supplementation. Trop. Anim. Health Prod., 28: 298-306.

29. NENGOMASHA E.M., 1997. The donkey (Equus asinus) as a draught animal in smallholder farming areas of the semi-arid regions of Zimbabwe. PhD thesis, CTVM, University of Edinburgh, UK.

30. NICHOLSON M.J., BUTTERW ORTH M., 1986. A guide to condition scoring of zebu cattle. Addis Ababa, Ethiopia, ILCA.

31. PEARSON R.A., LAWRENCE P.R., 1992. Intake, digestion, gastrointestinal transit time and nitrogen balance in working oxen: studies in Costa Rica and Nepal. Anim. Prod., 55: 361-370.

32. PEREZ R., VALENZUELA S., MERINO V., CABEZAS L., GARCIA M., BOU R., ORTIZ P., 1996. Energetic requirements and physiological adaptation of draught horses to ploughing work. Anim. Sci., 63: 343-351.

33. PRASAD V.L., MAROVANIDZE K., NYATHI P., 1991. The use of donkeys as draught animals relative to bovines in the communal farming sector of Zimbabwe. In: D. Fielding, R.A. Pearson eds., Donkeys, mules and horses in tropical agricultural development. Edinburgh, UK, University of Edinburgh, p. 231-239.

34. ROSE R.J., 1986. Endurance exercise in the horse - a review I, II. Br. vet. J., 142: 532-552.

35. SAMUI K.L., HUGH-JONES M.E., 1990. The financial and production impacts of bovine dermatophilosis in Zambia. Vet. Res. Commun., 14: 357-365.

36. SLIN GERLAND M.A., 1989. Selection of animals for work in SubSaharan Africa: research at the ICRISAT Sahelian Centre. In: D. Hoffmann, J. Nari, R.J. Petheran eds., Draught animals in rural development. Canberra, Australia, ACIAR, p. 203-210. (Proc. series No. 27)

37. STARKEY P.H., 1994. A world-wide view of animal traction highlighting some of the key issues in eastern and southern Africa. In: P. Starkey, E. M wenya, J. Stares eds., Improving animal traction technology. Proc. 1st Work. Animal Traction N etwork for Eastern and Southern Africa, Lusaka, Zambia, January 18-23, 1994. Wageningen, The N etherlands, CTA, p. 6-81.

38. TELENI E., 1993. Energy expenditure and nutrient requirement of working animals. In: W.J. Pryor ed., Draught animal power in the AsianAustralasian region. Work. in conjunction with 6th Asian-Australasian Assoc. Anim. Prod. Soc. Congr., Bangkok, Thailand, November 23-28, 1992. Canberra, Australia, ACIAR, p. 93-99. (Proc. N o. 46)

39. THOMAS C.K., PEARSON R.A., 1986. Effects of ambient temperature and head cooling on energy expenditure, food intake and heat tolerance of Brahman and Brahman x Friesian cattle working on treadmills. Anim. Prod., 43: 83-90.

40. TOPPS J.H., OLIVER J., 1993. Animal foods of Central Africa, 2nd ed., Technical handbook. Harare, Zimbabwe, Zimbabwe Agricultural Journal.

41. UPADHYAY R.C., MADAN M.L., 1985. Studies on blood acid-base status and muscle metabolism in working bullocks. Anim. Prod., 40: 11-16.

42. VALETTE J.P., BEDU S., 1990. Etude de la récupération cardiaque chez des chevaux trotteurs à l'entraînement. Equathlon, 8 : 19-23.

43. VALL E., 1996. Capacités de travail, comportement à l'effort et réponses physiologiques du zébu, de l'âne et du cheval au NordCameroun. Thèse doct., ENSAM, M ontpellier, France.

44. YOU SEF M.K., DILL D.B., FREELAND D.V., 1972. Energetic cost of grade walking in man and burro, Equus asinus: desert and mountain. J. Appl. Physiol., 33: 337-340. 


\section{Summary}

Pearson R.A., Vall E. Performance and management of draught animals in agriculture in Sub-Saharan Africa: A review

U se of animal power generally enables farmers in subSaharan Africa to increase agricultural production and improve the quality of life. Effective use of working animals depends on an understanding of the capabilities of the animals for work, their husbandry requirements and the factors which can influence their performance. These issues are reviewed in this paper in the context of the use of animal power in agriculture in Sub-Saharan Africa. Type of animal used for work determines power available to the farmer. The performance of donkeys, horses and cattle have been compared in work tests. Equids are more suited to rapid low draught activities where their faster speed can be used to advantage. At higher draught forces, where speed is less important, the additional weight and power of cattle are an advantage. U se of heart rate recovery after work gives a reasonable indication of fatigue and fitness of equids, when test conditions are standardized. Although feed requirements for work are generally low, feed quality can be so poor that animals are unable to eat sufficiently to meet energy needs for work, and lose weight during the work season. However, improvements in work performance are not always seen following supplementary feeding in the dry season and the economics need to be considered in each case. Food availability, diseases and heat stress, the major constraints to performance of draught cattle and donkeys working in SubSaharan Africa, are discussed.

Key words: Cattle - Ass - Horse - Draught animal - Evaluation W ork capacity - Animal performance - Livestock management Africa South of Sahara.

\section{Resumen}

Pearson R.A., Vall E. Rendimiento y manejo de animales de tiro en la agricultura del Africa sub-sahariana : una síntesis

El uso de la fuerza animal permite, en general, incrementar la producción agrícola y mejorar la calidad de vida de los agricultores del Africa sub-sahariana. El uso efectivo de los animales de trabajo depende de un conocimiento de su capacidad para el mismo, de sus condiciones de cría y de factores que influyen en su rendimiento. Este articulo revisa estos aspectos en un contexto del uso de la fuerza animal en la agricultura del Africa sub-sahariana. El tipo de animal usado para un determinado trabajo determina la fuerza de la que el agricultor dispone. El rendimiento de burros, caballos y bueyes (bóvidos) es comparado en dos pruebas de fuerza. Los équidos son más apropiados para actividades de tiro rápidas y ligeras, en las que su velocidad puede ser usada como ventaja. Para fuerzas mas pesadas, donde la velocidad es menos importante, el peso y la fuerza adicional de los bueyes (bóvidos) son una ventaja. El uso de la capacidad de recuperación cardíaca después del trabajo es un indicador razonable del grado de fatiga y de la condición física de los équidos, siempre y cuando dichas pruebas estén estandarizadas. A pesar de que los requerimientos alimenticios para el trabajo son por lo general bajos, su calidad puede ser tan pobre que los animales no consumen lo suficiente como para cubrir las necesidades energéticas que el trabajo requiere, por lo que pierden peso durante la estación de mayor actividad. Sin embargo, la suplementación durante la estación seca no siempre mejora el rendimiento, por lo que un análisis económico de la situación debe considerarse en cada caso particular. La disponibilidad de alimento, enfermedades y el estrés por calor, las mayores limitaciones del rendimiento de bueyes bóvidos y burros de tiro en Africa sub-sahariana, son discutidas.

Palabras clave: Ganado bovino - Asno - Caballo - Animal de tiro - Evaluación - Capacidad de trabajo - Desempeño animal - Manejo del ganado - Africa al sur del Sahara. 